\title{
Microstructural Effects on Damage Evolution in Shocked Copper Polycrystals
}

\author{
Evan J. Lieberman ${ }^{1,2}$, Ricardo A. Lebensohn ${ }^{2 *}$, David B. Menasche ${ }^{1}$, Curt A. Bronkhorst ${ }^{2}$, \\ Anthony D. Rollett ${ }^{1}$ \\ ${ }^{1}$ Carnegie Mellon University, 5000 Forbes Avenue, Pittsburgh, PA 15213, USA \\ ${ }^{2}$ Los Alamos National Laboratory, Los Alamos, NM 87545, USA
}

\begin{abstract}
Three-dimensional crystal orientation fields of a copper sample, characterized before and after shock loading using High Energy Diffraction Microscopy, are used for input and validation of direct numerical simulations using a Fast Fourier Transform (FFT)-based micromechanical model. The locations of the voids determined by X-ray tomography in the incipiently-spalled sample, predominantly found near grain boundaries, were traced back and registered to the preshocked microstructural image. Using FFT-based simulations with direct input from the initial microstructure, micromechanical fields at the shock peak stress were obtained. Statistical distributions of micromechanical fields restricted to grain boundaries that developed voids after the shock are compared with corresponding distributions for all grain boundaries. Distributions of conventional measures of stress and strain (deviatoric and mean components) do not show correlation with the locations of voids in the post-shocked image. Neither does stress triaxiality, surface traction or grain boundary inclination angle, in a significant way. On the other hand, differences in Taylor factor and accumulated plastic work across grain boundaries do correlate with the occurrence of damage. Damage was observed to take place preferentially at grain boundaries adjacent to grains having very different plastic response.
\end{abstract}

Keywords: Polycrystal Plasticity Modeling, Micromechanics, Damage Initiation, Deformation Inhomogeneities, Shocks.

\footnotetext{
* Corresponding author: Ricardo A. Lebensohn, Materials Science and Technology Division, Los Alamos National Laboratory, MS G755, Los Alamos, NM 87845, USA. Phone \#: +1-505-6653035, Fax \#: +1-505-667-8021, e-mail: lebenso@lanl.gov
} 


\section{1 - Introduction}

Predicting the conditions that determine material failure is one of the most fundamental challenges in materials science. One important failure mechanism for ductile materials undergoing dynamic loading conditions is void nucleation, growth and coalescence ${ }^{[1-8]}$. In the first stage, void nuclei under appropriate driving forces reach a size large enough to become stable and eventually sustain growth. At this nucleation phase of damage the influence of the material's microstructure can be very strong. Growth occurs when the volume of stable voids increases driven by stress triaxiality ${ }^{[2]}$, which reaches high values under the dynamic loading conditions considered in this work. Coalescence occurs when voids grow enough such that stress and strain concentrations in the surrounding material start overlapping and further increasing, promoting neighboring voids to join together ${ }^{[1]}$. It is at this stage in the process where ultimate failure of the material is initiated. The ability to predict the extent to which a given microstructure is vulnerable to damage nucleation and growth is relevant to the emerging field of computational materials design, to enable the discovery and development of new processes and/or materials with higher resistance to damage. The determination of whether or not voids will nucleate and grow in specific locations requires identifying which microstructural features promote or inhibit damage initiation.

Identifying potential microstructural features that influence damage requires knowledge of where damage develops within a microstructure under certain mechanical loading, and what is the micromechanical response at those locations. The use of non-destructive three-dimensional (3-D) imaging techniques such as near-field high-energy diffraction microscopy (nf-HEDM) ${ }^{[9-11]}$ and X-ray tomography, before and after deformation is applied, is ideal so that comparisons to the pristine sample can be made. Micromechanical fields can be obtained performing full-field simulations with direct input from the 3-D image of the initial microstructure.

There have been many investigations on the effect of various microstructure features on void formation within polycrystalline materials, although experimental constraints have often limited the scope of these studies. Field et al. ${ }^{[12]}$, Diard et al. ${ }^{[13]}$, Fensin et al. ${ }^{[8]}$, and Yang et al. ${ }^{[14]}$ analyzed multiple two-dimensional micrographs of damaged materials and found that damage preferentially occurred at interfaces with specific relationships to the principal loading directions, although the nature of the relationship depends on the type of loading (e.g. fatigue versus shock). 
The nature of the plastic response of the crystals vicinal to voids has also been associated with damage initiation by Wright et al. ${ }^{[15]}$, Bieler et al. ${ }^{[16,17]}$, Semiatin et al. ${ }^{[18]}$, Escobedo et al. ${ }^{[19,20]}$ and Yang et al. ${ }^{[14]}$, although different authors often made an arbitrary choice of parameters to correlate with damage, and the number of void sites studied was limited. The factors most frequently used to quantify plastic response are Schmid factor and Taylor factor.

In this work we study damage initiated by shock loading ${ }^{[19-24]}$. For shocked fcc materials, there has been a special focus on understanding the effect of $\Sigma 3$ boundaries with $\{111\}$ boundary normals, which are variously known as coherent twin boundaries or annealing twins. Escobedo et al. ${ }^{[19]}$, Fensin et al. ${ }^{[25]}$ and Yang et al. ${ }^{[14]}$ have shown, based on orientation maps of crosssections, that $\Sigma 3$ boundaries tend to be resistant to void formation. Although this suggests a resistance to damage based solely on interface character, the results presented here implicate heterogeneous plastic deformation.

Shock loading is frequently applied in gas-driven plate impact experiments, which provide a controlled method of developing a shock wave of a certain magnitude and thus testing dynamic damage processes ${ }^{[19,26-28]}$. Void nucleation occurs as part of the process of spallation failure. As the compressive wave propagates through the material, it eventually reflects off the rear surface of the sample, opposite to the side of initial impact, and returns as a rarefaction wave. This rarefaction wave then interacts with a similar reflected wave coming from the contact side of the sample (originating from compression of the impact plate) to form a region of high tensile stress at the spall plane. Full spallation occurs when the sample separates into two pieces at the spall plane. When the tensile stress is high enough to nucleate voids but not high enough to cause coalescence and full spallation, incipient spallation occurs, which is particularly useful for investigating early stages of porosity evolution.

In this work, we utilize data from an experiment in which a polycrystalline copper sample was characterized with nf-HEDM and then subjected to incipient spall. The recovered sample was again orientation-mapped using nf-HEDM and X-ray tomography. Registration of the 3-D images before and after the experiment allowed the locations of the eventual void sites to be established within the image of the undeformed sample, as described in Section 2. In turn, the undeformed microstructural image was used as input of a full-field crystal plasticity Fast Fourier Transform (FFT)-based model ${ }^{[29,30]}$ to simulate the micromechanical fields developed during 
the shock, as described in Section 3. In Section 4, we provide details of a novel technique for quantifying grain boundary and triple junction morphology directly from microstructural images, needed for subsequent analysis. In Section 5 we compare the predicted field distributions computed for the entire microstructure with those obtained only in the vicinity of regions developing porosity. This comparison allows us to assess the influence of different micromechanical fields on the occurrence of void nucleation and early growth, as discussed in Section 6.

\section{2 - Materials and Methods}

To investigate microstructural properties influencing damage initiation, this work utilizes the results of an experiment performed by Bingert et al. ${ }^{[31]}$ and analyzed by Menasche et al. ${ }^{[32]}$, whose goal was to obtain 3-D images of a material in which incipient spall occurred. In this experiment, a $99.997 \%$ pure polycrystalline copper sample was machined into a $1.2 \mathrm{~mm}$ diameter cylinder of height $2.42 \mathrm{~mm}$. This sample was then characterized using nf-HEDM ${ }^{[33]}$ over a height of $0.704 \mathrm{~mm}$. The sample was machined down to a $0.725 \mathrm{~mm}$ tall piece centered about the characterized section. This sample was then embedded into two copper polycrystal radial momentum traps and impacted by a copper flyer plate at $300 \mathrm{~ms}^{-1}$. The experimental setup involved placing the ringed sample onto the front of a pipe that was slightly larger in diameter than the HEDM characterized sample; thus when the target was impacted, the sample alone was stripped from the momentum trapping rings and soft-captured. The velocity was specifically selected such that the sample was recovered while causing incipient spall voids. The recovered sample was then characterized using both nf-HEDM and X-ray tomography. The end result was a pair of 3-D orientation maps of the same volume of the copper sample both before the experiment and after the plate impact had produced voids within the sample. Computed tomography also provided 3-D images of the porosity distribution. The tomography provided higher spatial resolution of the voids compared to the nf-HEDM image.

Menasche et $a{ }^{[32]}$ analyzed the experiment by mapping the voids characterized from the deformed sample onto the microstructure measured from the undeformed sample. First, a technique was developed to map the voids identified via X-ray tomography in the post-shocked sample to the corresponding nf-HEDM image. This was achieved by aligning larger-scale surface features present in both sample images. Next, the nf-HEDM images of the sample both 
undeformed and deformed were aligned with each other through the use of rotations and affine transformations. The purpose of this was to account for differences in sample alignment of the two measurements, as well as for the plastic deformation experienced by the sample as a result of the shock loading, which was on the order of a few percent in the spall plane region. This plastic deformation also made registration between the two nf-HEDM datasets more difficult because defect accumulation and crystal rotation results in larger uncertainty in crystal orientation. In more detail, the centers of mass of 1,800 grains were compared between the undeformed state and the post-shock state, of which approximately 1,000 were interior grains. The final result was that it was possible to identify certain regions within the nf-HEDM image of the undeformed sample as having developed voids in the deformed sample.

Using the orientation field of the undeformed sample obtained by Menasche et al. ${ }^{[32]}$ as the input of a micromechanical simulation, this work analyzes the local response of the material in the regions developing porosity. A full-field crystal plasticity model that utilizes FFT to solve the micromechanical governing equations ${ }^{[29,30]}$ is used to determine the local micromechanical fields under shock loading conditions, as described in the following section. Menasche et al. ${ }^{[32]}$ found that (see Table 1) of the 485 measured voids, 332 occurred at grain boundaries. Of those 332 grain boundary voids, 196 occurred on grain boundary planes, 126 occurred on triple junctions and 10 occurred on higher-order intersection points. Thus, grain boundaries regions will be the particular focus of our analysis because of the predominance of voids forming at those regions. Moreover, a number of micromechanical fields can be investigated at grain boundaries with the available modeling tools. Some of the fields that are unique to boundaries require the ability to quantitatively evaluate the interface normals. Novel techniques based on Cartesian moments are used to generate such values from the discrete microstructure images obtained by nf-HEDM.

\section{3 - Micromechanical Model}

Plastic deformation in high-purity polycrystalline copper considered in this study occurs at the single crystal level primarily by dislocation glide. Copper is an fcc material that deforms plastically by $\{111\}<110>$ slip. Polycrystals are aggregates of many single crystal grains, each having its own orientation, and the differences in orientation between neighbor grains leads to inhomogeneous distributions of stress and strain in the material. To understand the origin of failure in polycrystalline materials, which is a highly localized phenomenon, the local 
mechanical response the material must be studied at the same scale of damage events ${ }^{[34,35]}$. As previously mentioned, we use an infinitesimal strain elasto-viscoplastic Fast Fourier Transformbased (EVPFFT) model ${ }^{[30]}$ to compute the full-field micromechanical response directly from the voxelized images of the pre-shocked $\mathrm{Cu}$ polycrystal microstructure obtained by nf-HEDM. Note that for our analysis the micromechanical fields are calculated at the point of maximum tensile stress, for which the accumulated plastic strain is less than $3 \%$, justifying the use of a small strain approximation.

EVPFFT is based on the method developed by Moulinec and Suquet ${ }^{[36]}$ and improved by Michel et al. ${ }^{[37]}$ to predict the micromechanical behavior of linear and non-linear composites. Lebensohn et al. ${ }^{[30]}$ adapted the latter to polycrystal plasticity. The FFT-based model operates on a discrete grid of points representing a regularly-spaced sampling of the material's properties, such as a microstructure image measured by nf-HEDM, and calculates the values of the stress and strain fields at each grid point that fulfill equilibrium, compatibility and the constitutive relation, under given boundary conditions. The small-strain EVPFFT model uses a combined elastic and viscoplastic constitutive description that incorporates linear elasticity and an Euler implicit time discretization given by:

$$
\boldsymbol{\varepsilon}(x)=\varepsilon^{e}(x)+\varepsilon^{p}(x)=\boldsymbol{C}^{-1}(x): \boldsymbol{\sigma}(x)+\varepsilon^{p, t}(x)+\dot{\varepsilon}^{p}(x, \boldsymbol{\sigma}) \Delta t
$$

The strain tensor at each point $(\varepsilon)$ is calculated as the sum of the elastic strain $\left(\varepsilon^{\mathrm{e}}\right)$ and plastic strain $\left(\varepsilon^{\mathrm{p}}\right)$. The elastic strain is expressed via Hooke's Law as the inner product of the compliance tensor $\left(\mathbf{C}^{-1}\right)$ and Cauchy stress tensor $(\boldsymbol{\sigma})$. The plastic strain is expressed as a sum of the plastic strain which has accumulated up to time $t\left(\varepsilon^{\mathrm{p}, \mathrm{t}}\right)$ plus a viscoplastic strain increment associated with the viscoplastic strain rate, given by:

$$
\dot{\boldsymbol{\varepsilon}}^{p}(x)=\dot{\gamma}_{o} \sum_{s=1}^{N_{s}} \boldsymbol{m}^{s}(x)\left(\frac{\left|\boldsymbol{m}^{s}(x): \boldsymbol{\sigma}^{\prime}(x)\right|}{\tau^{s}(x)}\right)^{n} \times \operatorname{sgn}\left(\boldsymbol{m}^{s}(x): \boldsymbol{\sigma}^{\prime}(x)\right)
$$

where $\dot{\gamma}_{o}$ is a reference shear rate, $\mathrm{N}_{\mathrm{s}}$ is the total number of slip systems, $\mathbf{m}^{\mathrm{s}}$ is the symmetric Schmid tensor for slip system $s, \boldsymbol{\sigma}^{\prime}$ is the deviatoric stress tensor, $\tau^{\mathrm{s}}$ is the critical resolved shear stress (CRSS) of slip system $s$, and $n$ is the rate-sensitivity exponent. At the end of each step, the local CRSS and orientations are updated based on the calculated plastic slip. In this work we use a Voce hardening law to describe the evolution of CRSS via the equation: 


$$
\begin{aligned}
& \Delta \tau^{\alpha}=\frac{d \bar{\tau}^{\alpha}}{d \Gamma} \sum_{\beta} h^{\alpha \beta} \dot{\gamma}^{\beta} \\
& \bar{\tau}^{\alpha}=\tau_{0}+\left(\tau_{1}+\theta_{1} \Gamma\right)\left[1-\exp \left(-\frac{\theta_{0} \Gamma}{\tau_{1}}\right)\right]
\end{aligned}
$$

where $\tau^{\alpha}$ is the critical resolved shear stress on slip system $\alpha, \dot{\gamma}^{\beta}$ is the shear rate of slip on system $\beta, h^{\alpha \beta}$ is the latent hardening matrix that dictates how slip activity in different slip systems harden each other, $\Gamma$ is the accumulated slip across all slip systems and $\tau_{0}, \tau_{1}, \theta_{0}$, and $\theta_{1}$ are parameters associated with the Voce hardening law.

The presently available numerical implementation of the EVPFFT model cannot directly consider shock loading conditions. Therefore, we use the output of finite element (FE) simulations that model such conditions to provide input to the EVPFFT code. Here we use macro- and meso-scale FE simulations of the type reported by Bronkhorst et al. ${ }^{[38,39]}$. As mentioned previously, the spall plane is a region of high tensile stress cause by wave interactions and it is the effect of this tensile pulse that is considered in this work. In order to simulate microstructural effects of shock loading with EVPFFT, we need to obtain from FE simulations time-evolving strain-rate boundary conditions and Voce hardening parameters. For this, a FE simulation including a damage model described elsewhere ${ }^{[39]}$ was run for polycrystalline copper under shock loading conditions, to the point in time where the model predicted that damage initiation occurred. The sample geometry and loading type in this simulation represented the experiment by Bingert et al. ${ }^{[31]}$. This FE model predicted the time after impact at which void nucleation began, $0.15 \mu \mathrm{s}$, as well as the peak stresses at the spallation plane in the shock and radial directions, 944.77 $\mathrm{MPa}$ and 894.5 $\mathrm{MPa}$, respectively. Such values of stress indicate a large hydrostatic stress component with an overall tensile plastic response. Assuming a constant stress rate from 0 to the peak stress tensor, and discretizing the time from 0 to $0.15 \mu$ s in 299 steps, the strain rate at each time step was obtained. Figure 1a shows the resulting diagonal strain components as a function of time. The resulting sequence of strain rates was applied directly as the boundary conditions for the EVPFFT simulation. Note that plastic deformation continues to accumulate in the material after the point of peak tensile stress, especially in the vicinities of the pores, but this was not modeled here because the focus was on understanding microstructural effects on void initiation. The Voce hardening parameters were calibrated so as to reproduce the 
stress-strain response predicted by the FE model for polycrystalline copper. The result of this fit is shown in Fig. $1 \mathrm{~b}$ for the deviatoric and mean components of the stress. The mean square error between the FE and EVPFFT models for the von Mises stress-strain response is $1.31 \%$.

\section{4 - Triple Junction Analysis}

Since the voids in the analyzed sample predominately initiated at grain boundary features (grain boundaries, triple lines and quad points) it is necessary to quantify these features in the undeformed image. When working with microstructural images such as those provided by nfHEDM, the common method for quantifying surfaces is to generate a surface mesh of the image. However, the EVPFFT model works on a discrete grid of points, which is not directly compatible with a surface mesh and thus would necessitate interpolation between the mesh and the grid. In addition, the locations of the voids are also specified on the discrete nf-HEDM coordinate system and would require further interpolation to be related to the mesh. To perform this analysis effectively, we used a technique recently developed by Lieberman et al. ${ }^{[40]}$ that quantifies the grain interfaces directly on the nf-HEDM discrete grid, on which the EVPFFT model also operates. The technique is based on the calculation of the three unique first order Cartesian moments at each point near grain interfaces. These moments are proportional to the gradients of the grain interface along each Cartesian axis and so when rendered as a normalized vector, it becomes parallel to the local surface normal.

While the technique that utilizes first order Cartesian moments can define the grain boundary surface normals, it does not directly quantify the geometry of triple junctions. Menasche et al ${ }^{[32]}$ demonstrated that triple junctions are an important feature to consider in the present analysis. Accordingly, in order to analyze the triple junctions in addition to grain boundaries, we propose a technique that calculates the triple junction line vector using properties of second order Cartesian moments as described by Lichter and Dubowsky ${ }^{[41]}$. The second order Cartesian moments consist of six distinct values. These values are used to construct the diagonalizable symmetric matrix

$$
\left[\begin{array}{lll}
\mu_{200} & \mu_{110} & \mu_{101} \\
\mu_{110} & \mu_{020} & \mu_{011} \\
\mu_{101} & \mu_{011} & \mu_{002}
\end{array}\right]=P^{-1} \Lambda P
$$


where $\Lambda$ is a diagonal matrix composed of the eigenvalues of the second order Cartesian moment matrix and $\mathrm{P}$ is a matrix whose columns are the eigenvectors of the moment matrix. In this instance, the form of the discrete Cartesian moment equation used is as follows:

$$
\mu_{o p q}=\sum_{(i, j, k) \in S} w(\boldsymbol{r}) f(\boldsymbol{r})(i \Delta x)^{o}(j \Delta y)^{p}(k \Delta z)^{q}
$$

The indicator function $f(\mathbf{r})$ defines the shape under consideration by representing the points inside the shape as 1 and the points outside the shape as 0 . The weighting function $w(\boldsymbol{r})$ and volume of interest $S$ are defined through the application of a Gaussian weighting function. The purpose of the Gaussian weighting function is to effectively smooth the discrete data to a continuous function. The weighting function is defined as $w(\boldsymbol{r})=\exp \left[-\left(i^{2}+j^{2}+k^{2}\right) / \lambda^{2}\right]$. In this work the value of $\lambda$ is $\sqrt{ } 3$ and the extent of $S$ is spherical with a radius $r$ of 5.88 voxels, which is the distance for which the Gaussian weight is $10^{-5}$. If the indicator function used in the moment equation is representative of some object in space, the eigenvectors will correspond to the principal axes of that object while the eigenvalues are proportional to the relative magnitudes of size of the shape along the corresponding axes.

To make use of these properties, the triple junctions are expressed as voxelized shapes, each uniquely defined as the set of voxels that among their first nearest neighbors have the same three grain numbers. While in continuous 3-D space a triple junction is a one-dimensional feature, it becomes a cylindrical voxelized shape in a discrete 3-D space. These cylinders have a single major principal axis and two non-unique minor principal axes. Thus, the largest eigenvalue of the second order Cartesian matrix for a cylinder corresponds to the eigenvector that represents the major principal axis of that cylinder. When applied to a triple junction shape, this major principal axis is parallel to the triple junction line vector. An example of the results of this technique applied to a single triple junction is shown in Fig. 2. By using the two techniques of quantifying the grain boundary surface normals and triple junction tangent vectors we are able to analyze the morphological influence in void nucleation as well as calculate the surface tractions on these two features. 


\section{5 - Results}

The approximation of shock loading conditions described previously is applied to the voxelized microstructure image of the undeformed sample. For this, a unit cell must be properly constructed to perform the simulation. The rectangular grid of the nf-HEDM image has voxel dimensions of $768 \times 768 \times 64$ where the inter-voxel spacing is $2 \mu \mathrm{m}$ in the $\mathrm{x}$ - and $\mathrm{y}$-directions and $4 \mu \mathrm{m}$ in the z-direction. The resulting image is shown in Fig. 3(a). The next step is to construct a prismatic unit cell by embedding the nf-HEDM microstructure into a periodic polycrystalline unit cell. The purpose of this is two-fold: a) to represent the presence of the momentum trap rings used in the experiment, which were also polycrystalline copper with the same grain size, and b) provide the geometry and periodicity required by the EVPFFT model to operate upon. The polycrystalline microstructure surrounding the measured $\mathrm{Cu}$ sample was numerically generated using the Dream.3 $\mathrm{D}^{[42]}$ software package, and consists of randomly oriented FCC grains with an average grain size equivalent to that of the nf-HEDM microstructure. The combined microstructure image is shown in Fig. 3(b).

After applying the boundary conditions described in Section 2, the EVPFFT model gives the values of: Cauchy stress tensor, strain tensor, plastic strain rate tensor, updated orientation, Taylor factor and accumulated plastic energy density fields, for each voxel in the microstructure image. From the stress and strain tensors, their von Mises equivalents are obtained. Furthermore, with the mean stress also extracted from the stress tensor, the stress triaxiality (mean stress/von Mises stress) can be readily calculated. The Taylor factor is a scalar parameter that describes the ability of a given grain with a specific crystallographic orientation to accommodate the deformation to which it is subjected. Here the Taylor factor is calculated locally as follows ${ }^{[43]}$ :

$$
M(x)=\frac{\sum_{s=1}^{N^{s}}\left|\dot{\gamma}^{s}(x)\right|}{\dot{\varepsilon}_{v m}(x)}
$$

where $\dot{\gamma}^{s}(x)$ is the local shear rate on slip system $s, \mathrm{~N}^{s}$ is the total number of slip systems and $\dot{\varepsilon}_{v m}(x)$ is the local von Mises equivalent strain rate. In what follows, the analysis is performed on the Taylor factor field calculated at the final step of the simulation. The accumulated plastic work density represents the time integration of the rate of plastic work. This rate is calculated at each step of the EVPFFT simulation and is given by the following equation: 


$$
\dot{W}^{p}(x)=\boldsymbol{\sigma}(x): \dot{\varepsilon}^{p}(x)
$$

Using the grain boundary normal calculation technique described previously, along with the stress fields generated by the model, the grain boundary surface traction as well as its components are represented as:

$$
\begin{aligned}
& \vec{T}_{i}(x)=\sigma_{i j}(x) \hat{n}_{j}(x) \\
& T^{n}(x)=\vec{T}(x) \cdot \hat{n}(x) \\
& \vec{T}^{s}(x)=\vec{T}(x)-T^{n}(x) \hat{n}(x)
\end{aligned}
$$

where $\sigma_{i j}(\mathrm{x})$ is the local Cauchy stress tensor, $\hat{n}_{j}(\mathrm{x})$ is the local surface normal vector, $\mathrm{T}^{n}$ is the normal component of traction and $\mathrm{T}^{s}$ is the shear component of traction. Similar tractions can be calculated for the triple junctions, but this requires an additional step. The triple junction vector technique generates vectors that are tangent to the feature of interest rather than perpendicular, which is needed to describe a surface traction. Since a triple junction is a one-dimensional feature, there is not a unique perpendicular direction to it. Therefore, we choose to use the perpendicular vector that results in the largest normal component of traction as the one that represents the surface traction for the triple junctions:

$$
\vec{T}_{i}^{n}(x)=\max _{0 \leq \theta \leq 360}\left\{\sigma_{i j}(x)\left(R_{j k}(\theta) \hat{t}_{k}(x)\right)\right\}
$$

where $\hat{t}_{k}$ is one of the two unused principal axes (calculated from the eigenvectors of the second order moment matrix) that is perpendicular to the triple junction line vector, and $\mathrm{R}_{\mathrm{jk}}^{\theta}$ applies a rotation, $\theta$, around the triple junction line vector.

Mapping the locations of the voids onto the undeformed sample image requires the tomographic image of the voids. However, although the resolution of the tomographic image in the $\mathrm{x}$ - and $\mathrm{y}$ - directions is the same as the nf-HEDM image, the z-resolution of the tomography image is $1.5 \mu \mathrm{m}$ rather than $4 \mu \mathrm{m}$. The alignment established by Menasche et al. ${ }^{[32]}$ between the tomography of the deformed sample and the nf-HEDM of the undeformed sample allows voxels in both images to be measured by a unified set of coordinates established from the sample geometry. In order to register the void voxels measured by the tomography to the nf-HEDM image, we identify the nf-HEDM voxels that overlap the same physical space as the void voxels of the tomography. However, the difference in z-resolution between the images means that some 
voids will not be distinguishable from each other when they are registered to the nf-HEDM image, thus causing discrepancies in the void counts from Menasche et al. ${ }^{[32]}$. After the voids are rendered in the undeformed sample image (see Fig. 4), 447 voids are identified in the microstructure, with 308 of them being located on grain boundaries. Of those 308 grain boundary voids, 156 are located on grain boundary planes, 99 are located on triple junctions and 53 are located on higher order intersection points.

As shown in Table 1, while the overall ratio of grain boundary voids to total voids is the same as in Menasche et al. ${ }^{[32]}$, the breakdown of grain boundary voids is different. This is most extreme for the higher order intersection points and is due to the difference in z-resolution between the tomography and nf-HEDM image. The grain boundary classification of the voids is determined by counting the number of unique grains that are found in the set of voxels representing each void as well as the nearest neighbors of those void voxels. These additional voxels are considered to ensure that an accurate description of the region is accounted for. For example, there are cases where a void occurred on a grain boundary but grew preferentially into one grain versus the other; such a void may only be directly represented by voxels belonging to a single grain. To determine the influence of the different micromechanical fields described above on void initiation, discrete probability distributions of these fields are plotted for both all grain boundary voxels and for the grain boundary voxels associated with voids. The set of voxels representing the grain boundaries of the overall microstructure are limited to those within a certain sub-volume of the image both to represent the spallation region from the experiment and because of potential localization issues discussed in Menasche et al. ${ }^{[32]}$. This sub-volume is the set of voxels within layers 212 to 583 along the $x$-axis and 191 to 572 along the y-axis. The full length of the z-axis is included in this sub-volume. These dimensions correspond to the farthest extent at which the voids exist within the microstructure. Outside this region no voids were detected in the tomography. The set of voxels included in the analysis on grain boundary developing voids is limited to voxels that are identified as being grain boundary voxels. Within that set, a voxel is considered a part of the void analysis if it is labeled as a void voxel from the tomography information or is a neighbor to such a voxel.

In order to compare distributions of micromechanical fields for the full set of grain boundary voxels ("full" distributions) versus the corresponding distributions restricted to those voxels that 
developed porosity after the shock ("restricted" distributions) we quantify the difference between distributions using the Hellinger distance ${ }^{[44]}$, which is calculated as:

$$
d_{H}(U, V)=\frac{1}{\sqrt{2}} \sqrt{\sum_{i=1}^{k}\left(\sqrt{u_{i}}-\sqrt{v_{i}}\right)^{2}}
$$

where $U$ and $V$ are discrete probability distributions, $u_{i}$ and $v_{i}$ are the probability values for the $i^{\text {th }}$ bin of $U$ and $V$ respectively and $k$ is the total number of bins in the probability distribution. The reciprocal square root of two in the pre-factor ensures that the Hellinger distance has a range of zero to one. Over this range, a value of zero indicates that the two distributions are identical while a value of one indicates that there is zero overlap between the two distributions.

Next we evaluate correlations of porosity with micro-mechanical response. The simplest micromechanical fields relevant to this comparison are mean stress, von Mises stress, von Mises strain and stress triaxiality, which are directly calculated from the stress and strain tensors predicted by the EVPFFT model at every point in the material. The comparisons of the distributions of these fields are shown in Fig. 5. The violin plots display a smoothed representation, reflected with respect to the horizontal axis, of the discrete probability distribution with a boxplot displayed within. The edges of the grey boxplot correspond to the first and third quartiles, the black line shows the 1.5 interquartile range limits; the combination of solid circle and vertical line represents the median while the crossed circle represents the mean. The Hellinger distances for these fields are 0.082 for the hydrostatic stress, 0.068 for the von Mises stress, 0.075 for the von Mises strain, and 0.062 for the stress triaxiality. Consistent with these low values, there is no significant difference in the medians or spreads between the distributions; the most notable difference is how the upper tails of the stress triaxiality distributions differ.

The next field distributions considered are those associated grain boundary features, namely the normal component of the surface tractions and grain boundary inclination angles. For the surface traction distributions, values from both the grain boundary planes and the triple junction lines are represented together in Fig. 6, where the Hellinger distance is 0.066 . The grain boundary inclination angles are relative to the shock direction and have been separated between those for grain boundary planes and those for triple junctions as shown in Fig. 7 and have 
Hellinger distances of 0.036 and 0.024 respectively. Violin plots were not used because of the difficulty in rendering distributions that have finite set limits. None of these factors show much difference in the distributions for grain boundaries vicinal to pores versus those for all boundaries; likewise, the Hellinger distances are also small. Next, the local Taylor factor and plastic work are used to describe the contrast in plastic response of the grains on both sides of a boundary by finding the greatest absolute difference in the local values on both sides. The resulting distribution comparisons are shown in Fig. 8. The distributions of the Taylor factor difference have a Hellinger distance of 0.276 while the distributions of the plastic work difference have a Hellinger distance of 0.326. These values are well above zero and are noticeably larger than for any of the other distributions compared previously. There is also a noticeable difference between the medians for both micromechanical indicators with the entirety of the grain boundaries producing voids distributions being shifted to higher values compared to the full distributions.

Further granularity can be obtained by sub-dividing the distributions considered above based on the character of the grain boundaries involved. As described previously, there is particular interest in $\Sigma 3$ grain boundaries in copper polycrystals, thus the distributions shown previously are separated into distributions for $\Sigma 3$ boundaries and for all other boundaries. For this analysis, the boundaries that are counted as $\Sigma 3$ are those that are within $5^{\circ}$ of rotation of the specific $\Sigma 3$ misorientation of a $60^{\circ}$ around a $<111>$ crystal axis. The data are separated into distributions for the three different grain boundary types, which are then further separated into plots for void boundaries and for all boundaries, with the difference in Taylor factor shown in Fig. 9 and the difference in plastic work in Fig. 10. For the difference in Taylor factor, the Hellinger distance between $\Sigma 3$ boundaries and all other boundaries is 0.182 for the grain boundary void voxels and 0.136 for all grain boundary voxels. For the difference in plastic work, the Hellinger distance between $\Sigma 3$ boundaries and all other boundaries is 0.151 for the restricted distribution and 0.12 for the full distribution. For both distributions the $\Sigma 3$ boundaries are shifted towards slightly larger values, which can be interpreted as an indication of the boundary type being associated with greater plasticity differences for voiding. Another way of analyzing the significance of grain boundary type is to calculate Hellinger distances between the full and restricted distributions for the two grain boundary groups, rather than between the grain boundary groups within the full and restricted distributions separately, as shown previously. The resulting Hellinger distances, 
along with those found before the separation by grain boundary character, are shown in Table 2 . Both forms of comparison show that $\Sigma 3$ boundaries with voids have slightly greater differences than the other boundaries.

\section{6 - Discussion}

While the predicted stress and strain distributions shown in Fig. 5 have no correlations with the subsequent occurrence of void nucleation at grain boundaries, Fig. 8 indicates that the more elaborate measures of the mechanical response at the boundaries do. In effect, two-point correlation measures (differences in Taylor factor and plastic work), reveal more than one-point ones. There is also no correlation between grain boundary inclination to the shock direction and void nucleation as shown in Fig. 7, a result that is in contradiction to what has been found in other experiments mentioned previously. However, the unique size and scale of the sample and experiment might explain the different observations. Given that stress triaxiality already has been connected to damage processes in previous studies ${ }^{[2,45]}$, it is surprising that no correlation exists in this data set, Fig. 5(d). However, it cannot be determined if this is due to void nucleation or void growth, and it is the former that stress triaxiality is most closely associated with, e.g., in the necks of tensile samples. Note that stress triaxiality has been a useful quantity within macroscale analyses of porosity evolution but it is not clear how physically meaningful stress triaxiality is at the length scales of the single crystal. In other words, the results presented here show that, while high triaxiality may assist void formation, especially where fracture of second phase particles initiates voiding, e.g. Maire et al. ${ }^{[46]}$, local variations at the microstructure scale do not explain specific occurrences of voids.

The most interesting results presented above are those corresponding to differences in Taylor factor and plastic work across the boundaries. The differences between the full and restricted distributions is greater than for any other micromechanical fields, and not just a difference in shape but also a large difference in medians as well. What this implies is that damage tends to occur preferentially on grain boundaries that are interfaces between crystals that have a greater contrast in plastic response. As the material deforms, these highly contrasted grain pairs begin to experience greater differences in local stress and strain that eventually leads to incompatibility that promotes damage. Previous experimental works ${ }^{[14-20]}$ have found potential relationships between differences in Taylor factor and damage by measuring orientations, but differences in 
plastic work were not explored in this context, because micromechanical simulations are required to obtain this information. Taylor factor is a variable that can change with strain as the crystals rotate and harden with deformation, while plastic work is an accumulated value; despite this difference, the distributions of these magnitudes are comparable in this case, because relatively small plastic strains were involved.

While there is a clear difference between grain boundaries that developed voids and those that do not, the separation is not absolute; there are boundaries in other parts of the microstructure with similar contrast in plastic properties where no damage was found. One possible explanation is that the presence of damage depends on other factors in addition to plasticity contrast, such as the stress factors analyzed previous (i.e. von Mises stress and surface traction) or grain boundary character. These potential additional influences might only become revealed with in situ measurements that describe the order in which the voids nucleated. Another aspect to consider is that the EVPFFT model, like many crystal plasticity models, does not incorporate the influence of the grain boundary structure on the mechanical response. One example of such an influence is slip transmission; works by Bieler et al. ${ }^{[17,47]}$ discuss many of the influences of slip transmission on micromechanics.

In general, the $\Sigma 3$ boundaries on which voids occurred are shifted towards slightly higher differences in Taylor factor and plastic work when compared to both the rest of the grain boundaries as well as the $\Sigma 3$ boundaries that did not develop damage. Such a shift hints at the elevated resistance to damage of $\Sigma 3$ boundaries, which is also shown in other studies although the shift seen in the present analysis is rather small. These results do, however, agree with what was observed by Menasche et al. ${ }^{[32]}$ on this same data set, who found that there is no significant difference in the occurrence of damage on $\Sigma 3$ boundaries versus other boundary types, a trend that was also observed when considered here on the nf-HEDM image. However, the influence of grain boundary structure on the polycrystalline mechanical response is not yet sufficiently understood and therefore not yet implemented in EVPFFT, which precluded evaluation of its effect on the micromechanical field distributions in this instance.

\section{7 - Conclusion}


Three-dimensional crystal orientation fields of a polycrystalline copper sample characterized before and after shock loading using nf-HEDM were combined with micromechanical modelling with direct input from these fields to discover microstructural effects on damage evolution in the material. The shock caused incipient spallation with a consequent population of voids, most of which were adjacent to grain boundaries as determined by concurrent X-ray tomography of the post-shock material. By applying techniques developed by Menasche et al. ${ }^{[32]}$, the locations of the voids were registered against the 3-D orientation map of the undeformed sample. EVPFFT simulation assisted by FE analysis to provide boundary conditions consistent with shock loading were used to calculate the micromechanical fields developing during the shock at the peak stress, i.e. when damage initiated. Hellinger distance, which quantifies the difference between statistical distributions, was used to compare the distributions of various micromechanical fields for all grain boundaries against the restricted population of grain boundaries near which damage occurred as a consequence of the shock. Conventional measures of stress and strain (deviatoric and mean components) did not show correlation with damage initiation. Neither did stress triaxiality, surface traction or grain boundary inclination angle, even though previous evidence or speculation indicated that such factors could be relevant to determine porosity evolution at different stages of the ductile damage process. In this regard, it should be emphasized that the present analysis does not necessarily preclude the possibility of such factors affecting porosity evolution at later stages of damage evolution. On the other hand, differences in Taylor factor and accumulated plastic work across grain boundaries did correlate with the occurrence of damage in the incipient spall experiment. In particular, damage was observed to occur preferentially at grain boundaries between grains with very different plastic response.

\section{Acknowledgements}

The authors wish to thank Saryu Fensin, Edward Kober and John Bingert (LANL), and Robert Suter (CMU) for fruitful discussions. This work was supported by Los Alamos National Laboratory's Directed Research and Development (LDRD-DR Project 20140114DR). RAL and $\mathrm{CAB}$ also acknowledge support from the Joint DoD/DOE Munitions Technology Programs. ADR also acknowledges support from the US Department of Energy, National Nuclear Security Administration, under contract number DE-NA0002918. The data was collected at the Advanced 
Photon Source (beamline 1-ID), supported by the US Department of Energy, Office of Science, Office of Basic Energy Sciences under contract number DE-AC02-06CH11357.

\section{References}

[1] D.R. Curran, L. Seaman, D.A. Shockey. Dynamic failure of solids, Physics Reports 147 (1987) 253-388.

[2] W.M. Garrison Jr, N.R. Moody. Ductile fracture, Journal of Physics and Chemistry of Solids 48 (1987) 1035-1074.

[3] J. Gurland. Observations on the fracture of cementite particles in a spheroidized $1.05 \% \mathrm{c}$ steel deformed at room temperature, Acta Metallurgica 20 (1972) 735-741.

[4] F.M. Beremin. Cavity formation from inclusions in ductile fracture of A508 steel, MTA 12 (1981) 723-731.

[5] M.N. Shabrov, A. Needleman. An analysis of inclusion morphology effects on void nucleation, Modelling and Simulation in Materials Science and Engineering 10 (2002) 163.

[6] D. Hull, D.E. Rimmer. The growth of grain-boundary voids under stress, Philosophical Magazine 4 (1959) 673-687.

[7] D.P. Field, B.L. Adams. Interface cavitation damage in polycrystalline copper, Acta Metallurgica et Materialia 40 (1992) 1145-1157.

[8] S.J. Fensin, J.P. Escobedo-Diaz, C. Brandl, E.K. Cerreta, G.T. Gray Iii, T.C. Germann, S.M. Valone. Effect of loading direction on grain boundary failure under shock loading, Acta Materialia 64 (2014) 113-122.

[9] R.M. Suter, D. Hennessy, C. Xiao, U. Lienert. Forward modeling method for microstructure reconstruction using x-ray diffraction microscopy: single-crystal verification, Review of Scientific Instruments 77 (2006) 123905-123901.

[10] R. Pokharel, S.F. Li, J. Lind, C.M. Hefferan, U. Lienert, R.A. Lebensohn, R.M. Suter, A.D. Rollett. Quantifying Damage Accumulation Using State-of-The-Art FFT Method, Materials Science Forum 702-703 (2012) 515-518.

[11] C.M. Hefferan, S.F. Li, J. Lind, U. Lienert, A.D. Rollett, P. Wynblatt, R.M. Suter. Statistics of high purity nickel microstructure from high energy X-ray diffraction microscopy, Computers, Materials and Continua 14 (2009) 207-217.

[12] D. Field, B. Adams. Heterogeneity of intergranular damage, MTA 23 (1992) 2515-2526.

[13] O. Diard, S. Leclercq, G. Rousselier, G. Cailletaud. Distribution of normal stress at grain boundaries in multicrystals: application to an intergranular damage modeling, Computational Materials Science 25 (2002) 73-84.

[14] Y. Yang, P. Zhi-qiang, C. Xing-zhi, G. Zhao-liang, T. Tie-gang, H. Hai-bo, Z. Qingming. Spall behaviors of high purity copper under sweeping detonation, Materials Science and Engineering: A 651 (2016) 636-645.

[15] S.I. Wright, D.P. Field. Recent studies of local texture and its influence on failure, Materials Science and Engineering: A 257 (1998) 165-170.

[16] T. Bieler, R.L. Goetz, S.L. Semiatin. Anisotropic plasticity and cavity growth during upset forging of Ti-6Al-4V, Materials Science and Engineering: A 405 (2005) 201-213.

[17] T.R. Bieler, P. Eisenlohr, C. Zhang, H.J. Phukan, M.A. Crimp. Grain boundaries and interfaces in slip transfer, Current Opinion in Solid State and Materials Science 18 (2014) 212226. 
[18] P.D. Nicolaou, S.L. Semiatin. An analysis of cavity growth during open-die hot forging of Ti-6Al-4V, Metallurgical and Materials Transactions A 36 (2005) 1567-1574.

[19] J.P. Escobedo, E.K. Cerreta, D. Dennis-Koller, C.P. Trujillo, C.A. Bronkhorst. Influence of boundary structure and near neighbor crystallographic orientation on the dynamic damage evolution during shock loading, Philosophical Magazine 93 (2012) 833-846.

[20] A.G. Perez-Bergquist, J.P. Escobedo, C.P. Trujillo, E.K. Cerreta, G.T. Gray, III, C. Brandl, T.C. Germann. The role of the structure of grain boundary interfaces during shock loading. Shock Compression of Condensed Matter - 2011: Conference of the American Physical Society Topical Group on Shock Compression of Condensed Matter, 26 June-1 July 2011, vol. 1426. USA: American Institute of Physics, 2012. p.1359-1362.

[21] J. Belak. On the nucleation and growth of voids at high strain-rates, Journal of ComputerAided Materials Design 5 (1998) 193-206.

[22] R.E. Rudd, J.F. Belak. Void nucleation and associated plasticity in dynamic fracture of polycrystalline copper: an atomistic simulation, Computational Materials Science 24 (2002) 148153.

[23] S. Christy, H.R. Pak, M.A. Meyers. Effects of Metallurgical Parameters on Dynamic Fracture by Spalling of Copper. Metallurgical Applications of Shock-Wave and High-StrainRate Phenomena. New York and Basel, 1986.

[24] J.P. Escobedo, D. Dennis-Koller, E.K. Cerreta, B.M. Patterson, C.A. Bronkhorst, B.L. Hansen, D. Tonks, R.A. Lebensohn. Effects of grain size and boundary structure on the dynamic tensile response of copper, Journal of Applied Physics 110 (2011) 033513 (033513 pp.).

[25] S.J. Fensin, E.K. Cerreta, G.T.G. Iii, S.M. Valone. Why are some Interfaces in Materials Stronger than others?, Sci. Rep. 4 (2014).

[26] L.S. T. Antoun, D. Curran, G. Kanel, S. Razorenov, A. Utkin. Spall Fracture, Springer, New York, 2002.

[27] M.A. Meyers. Dynamic behavior of materials, John Wiley \& Sons, 1994.

[28] G.T. Gray, III, L.M. Hull, V. Livescu, J.R. Faulkner, M.E. Briggs, E.K. Cerreta. Influence of sweeping detonation-wave loading on shock hardening and damage evolution during spallation loading in tantalum. DYMAT 2012 - 10th International Conference on the Mechanical and Physical Behaviour of Materials under Dynamic Loading, 2-7 Sept. 2012, vol. 26. France: EDP Sciences, 2012. p.02004 (02006 pp.).

[29] R.A. Lebensohn. N-site modeling of a 3D viscoplastic polycrystal using Fast Fourier Transform, Acta Materialia 49 (2001) 2723-2737.

[30] R.A. Lebensohn, A.K. Kanjarla, P. Eisenlohr. An elasto-viscoplastic formulation based on fast Fourier transforms for the prediction of micromechanical fields in polycrystalline materials, International Journal of Plasticity 32-33 (2012) 59-69.

[31] J. Bingert, R. Suter, J. Lind, S. Li, R. Pokharel, C. Trujillo. High-Energy Diffraction Microscopy Characterization of Spall Damage. in: Song B, Casem D, Kimberley J, (Eds.). Dynamic Behavior of Materials, Volume 1. Springer International Publishing, 2014. pp. 397403.

[32] D.B. Menasche, J. Lind, S.F. Li, P. Kenesei, J.F. Bingert, U. Lienert, R.M. Suter. Shock induced damage in copper: A before and after, three-dimensional study, Journal of Applied Physics 119 (2016) 154902.

[33] S.F. Li, R.M. Suter. Adaptive reconstruction method for three-dimensional orientation imaging, Journal of Applied Crystallography 46 (2013) 512-524. 
[34] S.R. Kalidindi, A. Bhattacharyya, R.D. Doherty. Detailed analyses of grain-scale plastic deformation in columnar polycrystalline aluminium using orientation image mapping and crystal plasticity models, Proceedings of the Royal Society of London. Series A: Mathematical, Physical and Engineering Sciences 460 (2004) 1935-1956.

[35] N. Allain-Bonasso, F. Wagner, S. Berbenni, D.P. Field. A study of the heterogeneity of plastic deformation in IF steel by EBSD, Materials Science and Engineering: A 548 (2012) 5663.

[36] H. Moulinec, P. Suquet. A numerical method for computing the overall response of nonlinear composites with complex microstructure, Computer Methods in Applied Mechanics and Engineering 157 (1998) 69-94.

[37] J.C. Michel, H. Moulinec, P. Suquet. A computational method based on augmented Lagrangians and fast Fourier transforms for composites with high contrast, Computer Modeling in Engineering \&amp; Sciences 1 (2000) 79-88.

[38] C.A. Bronkhorst, B.L. Hansen, E.K. Cerreta, J.F. Bingert. Modeling the microstructural evolution of metallic polycrystalline materials under localization conditions, Journal of the Mechanics and Physics of Solids 55 (2007) 2351-2383.

[39] C.A. Bronkhorst, G.T. Gray, F.L. Addessio, V. Livescu, N.K. Bourne, S.A. MacDonald, P.J. Withers. Response and Representation of Ductile Damage under Varying Shock Loading Conditions in Tantalum, Journal of Applied Physics 119 (2016).

[40] E.J. Lieberman, A.D. Rollett, R.A. Lebensohn, E.M. Kober. Calculation of grain boundary normals directly from 3D microstructure images, Modelling and Simulation in Materials Science and Engineering 23 (2015) 035005.

[41] M.D. Lichter, S. Dubowsky. Estimation of state, shape, and inertial parameters of space objects from sequences of range images. Intelligent Robots and Computer Vision XXI:

Algorithms, Techniques, and Active Vision, 28 Oct. 2003, vol. 5267. USA: SPIE-Int. Soc. Opt. Eng., 2003. p.199-210.

[42] M. Groeber, M. Jackson. DREAM.3D: A Digital Representation Environment for the Analysis of Microstructure in 3D, Integrating Materials and Manufacturing Innovation 3 (2014) 5 .

[43] C.N. Reid. Deformation geometry for materials scientists. [1st ed.] ed., Oxford, Pergamon Press, 1973.

[44] A.L. Gibbs, F.E. Su. On Choosing and Bounding Probability Metrics, International Statistical Review 70 (2002) 419-435.

[45] R.A. Lebensohn, J.P. Escobedo, E.K. Cerreta, D. Dennis-Koller, C.A. Bronkhorst, J.F. Bingert. Modeling void growth in polycrystalline materials, Acta Materialia 61 (2013) 69186932.

[46] E. Maire, O. Bouaziz, M. Di Michiel, C. Verdu. Initiation and growth of damage in a dual-phase steel observed by X-ray microtomography, Acta Materialia 56 (2008) 4954-4964.

[47] T.R. Bieler, P. Eisenlohr, F. Roters, D. Kumar, D.E. Mason, M.A. Crimp, D. Raabe. The role of heterogeneous deformation on damage nucleation at grain boundaries in single phase metals, International Journal of Plasticity 25 (2009) 1655-1683.

\section{Figure Captions}

Figure 1: (a) Strain history obtained with FE and applied to the EVPFFT simulation and (b) stress-strain curves for the FE and EVPFFT simulations. Solid lines represent von Mises stress 
and dashed lines represent hydrostatic stress. Note the different vertical scales, evidencing high triaxiality.

Figure 2: A set of voxels for a single triple line between three grains, determined by the number of unique grains amongst nearest neighbors. At each point of the triple line a local tangent vector is calculated and shown as an arrow glyph.

Figure 3: Orientation map of undeformed copper polycrystalline sample (a) measured via nfHEDM that was (b) embedded in a synthetic random copper polycrystal with approximately the same grain size.

Figure 4: The voxels representing voids formed in shock loading, in black, are displayed within the transparent orientation map of the nf-HEDM of the undeformed microstructure, viewed along the shock direction. Note that the voids form an annulus with varying density of voids around the ring.

Figure 5: Violin plots of the distributions of (a) mean stress, (b) von Mises stress, (c) von Mises strain and (d) stress triaxiality for both the overall set of grain boundaries and for the grain boundaries at the void locations.

Figure 6: Violin plots comparing surface normal tractions for all grain boundaries and for the grain boundaries where voids are located.

Figure 7: Comparison between all grain boundaries and grain boundaries developing porosity for: (a) the grain boundary plane normal inclination to shock direction and for (b) the triple junction tangent vector inclination to shock direction.

Figure 8: Violin plots of the distributions of (a) difference in Taylor factor and (b) difference in plastic work across a grain boundary for both the overall set of grain boundaries and for the grain boundaries at the void locations.

Figure 9: Violin plots for difference in Taylor factor partitioned by grain boundary type into sets for $\Sigma 3$ boundaries and all other boundaries and separately displayed for (a) the grain boundaries developing porosity and for (b) all grain boundaries.

Figure 10: Violin plots for difference in plastic work partitioned by grain boundary type into sets for $\Sigma 3$ boundaries and all other boundaries and separately displayed for (a) the grain boundaries developing porosity and for (b) all grain boundaries. 


\section{Table Captions}

Table 1: Distribution of voids at grain boundary features from Menasche et $a .^{[32]}$ and from this work.

Table 2: Hellinger distances between full and restricted distributions by grain boundary character. 


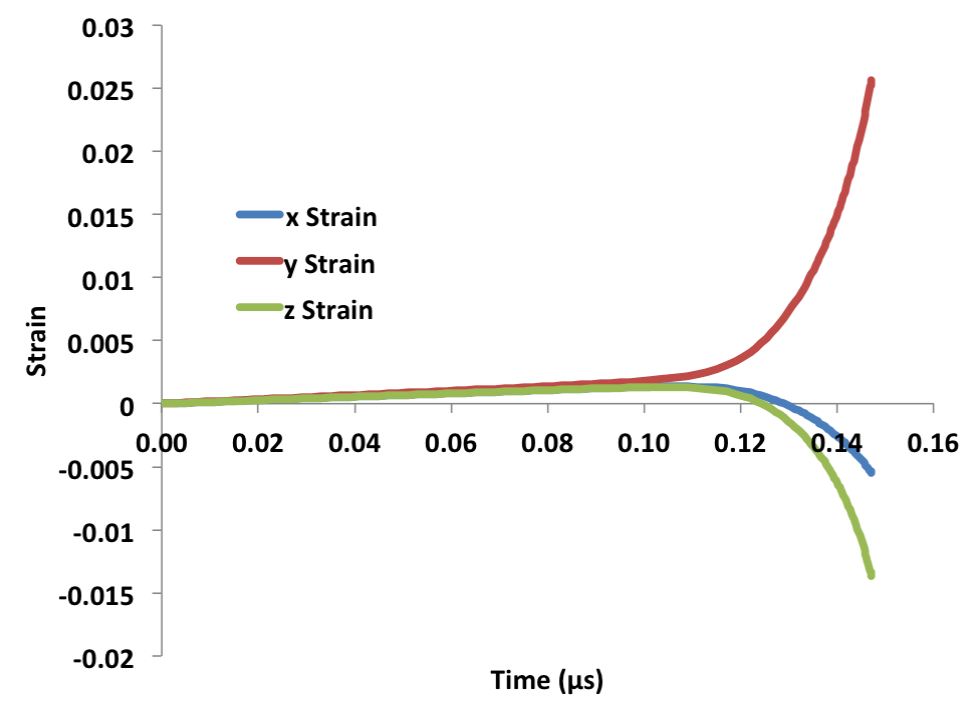

(a)

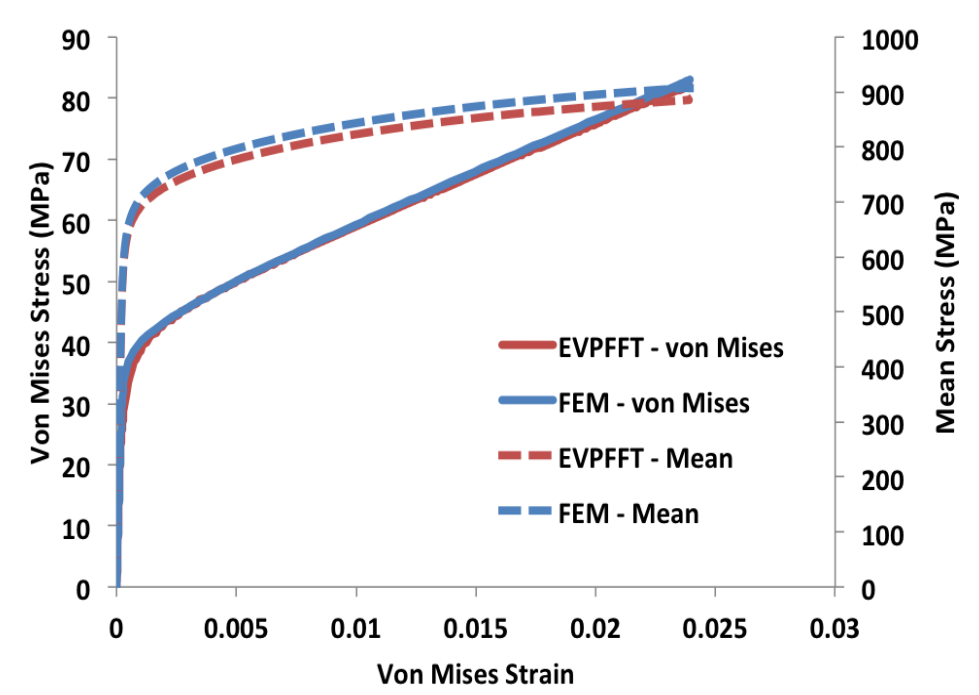

(b)

Figure 1: (a) Strain history obtained with FE and applied to the EVPFFT simulation and (b) stress-strain curves for the FE and EVPFFT simulations. Solid lines represent von Mises stress and dashed lines represent hydrostatic stress. Note the different vertical scales, evidencing high triaxiality. 


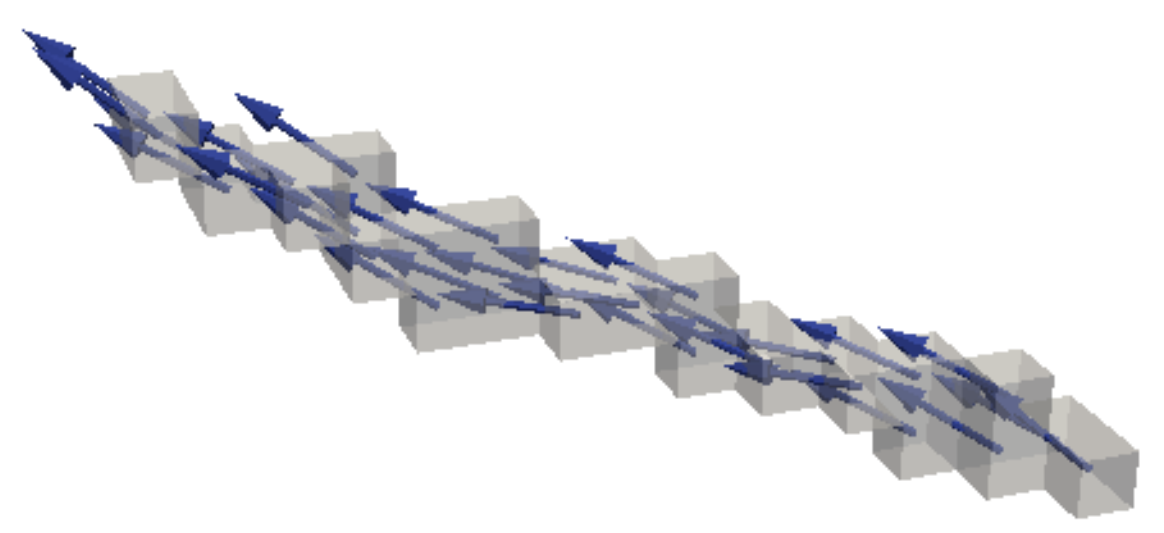

Figure 2: A set of voxels for a single triple line between three grains, determined by the number of unique grains amongst nearest neighbors. At each point of the triple line a local tangent vector is calculated and shown as an arrow glyph. 
(a)
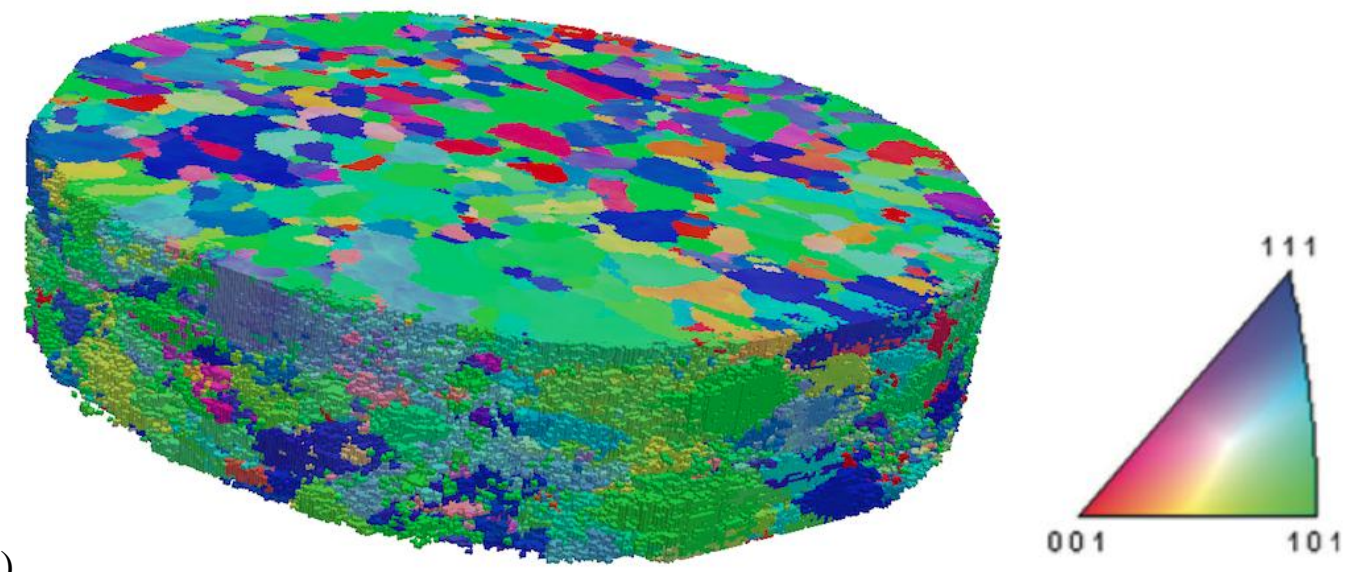

(b)

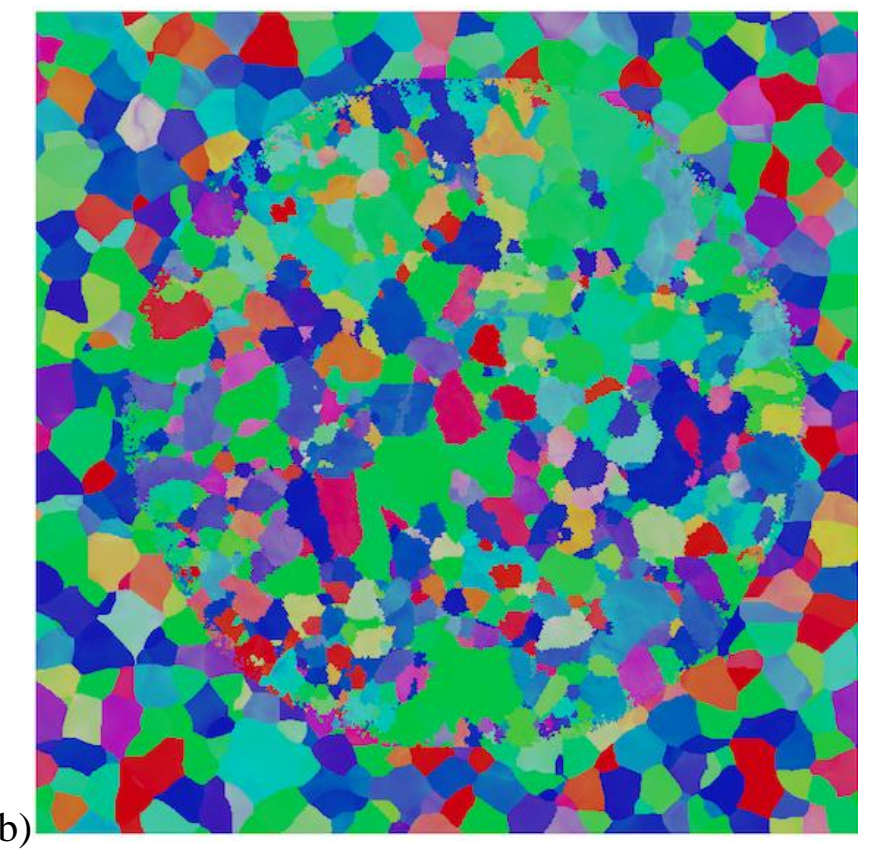

Figure 3: Orientation map of undeformed copper polycrystalline sample (a) measured via nf-HEDM that was (b) embedded in a synthetic random copper polycrystal with approximately the same grain size. 


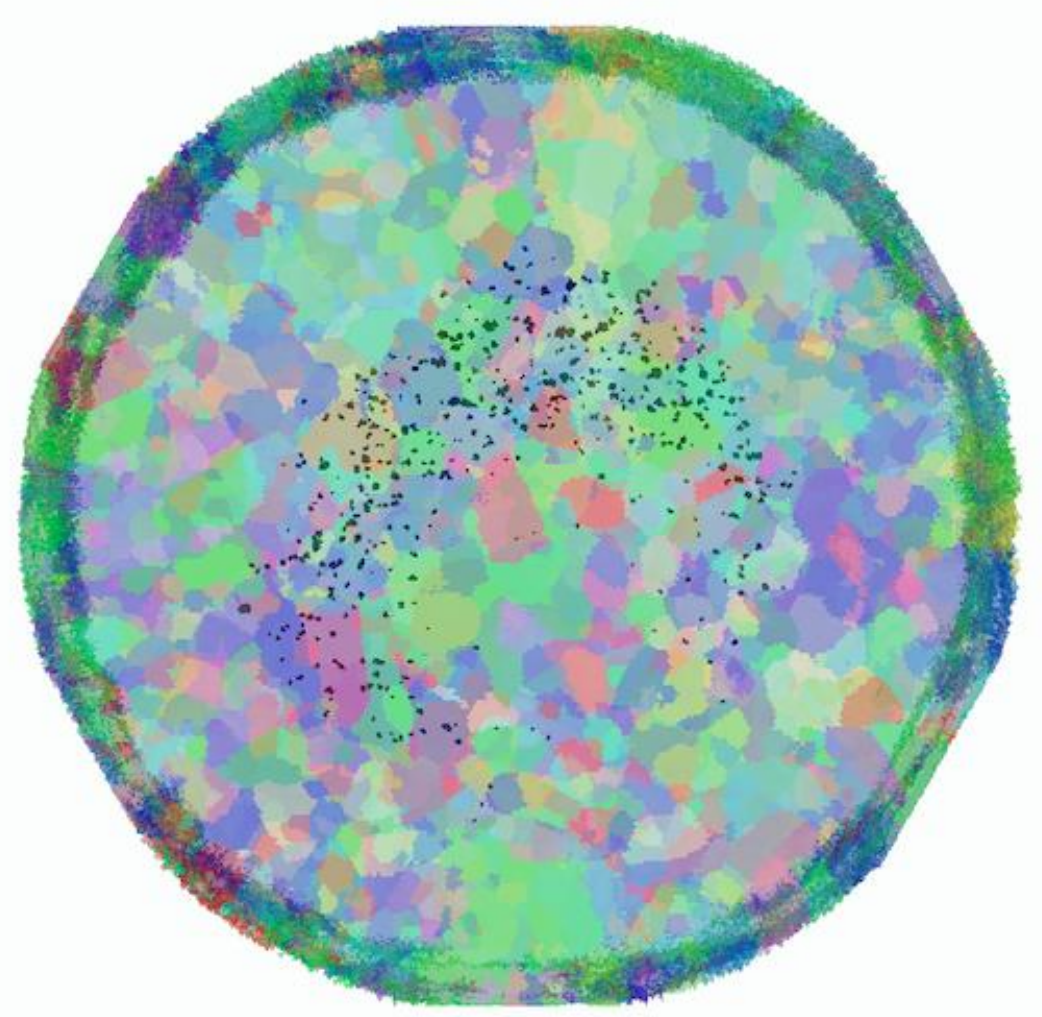

Figure 4: The voxels representing voids formed in shock loading, in black, are displayed within the transparent orientation map of the nfHEDM of the undeformed microstructure, viewed along the shock direction. Note that the voids form an annulus with varying density of voids around the ring. 

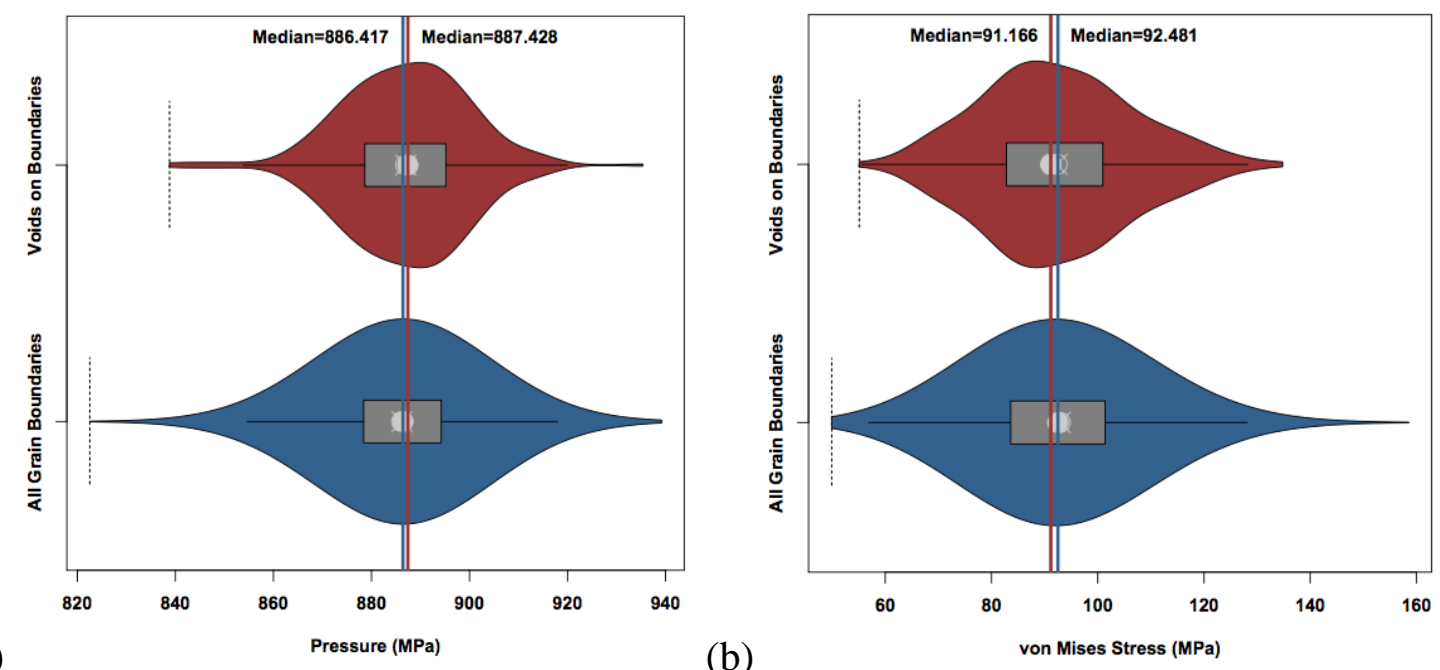

(a)

(b)
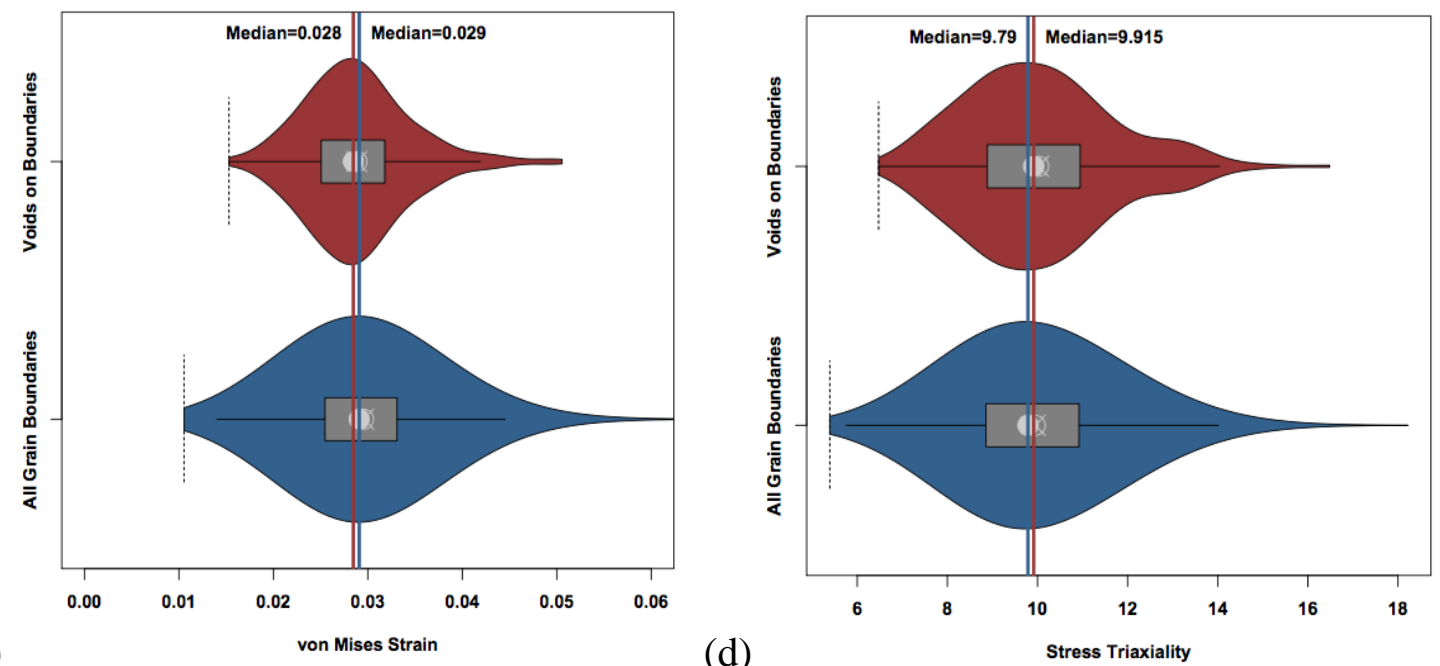

Figure 5: Violin plots of the distributions of (a) mean stress, (b) von Mises stress, (c) von Mises strain and (d) stress triaxiality for both the overall set of grain boundaries and for the grain boundaries at the void locations. 


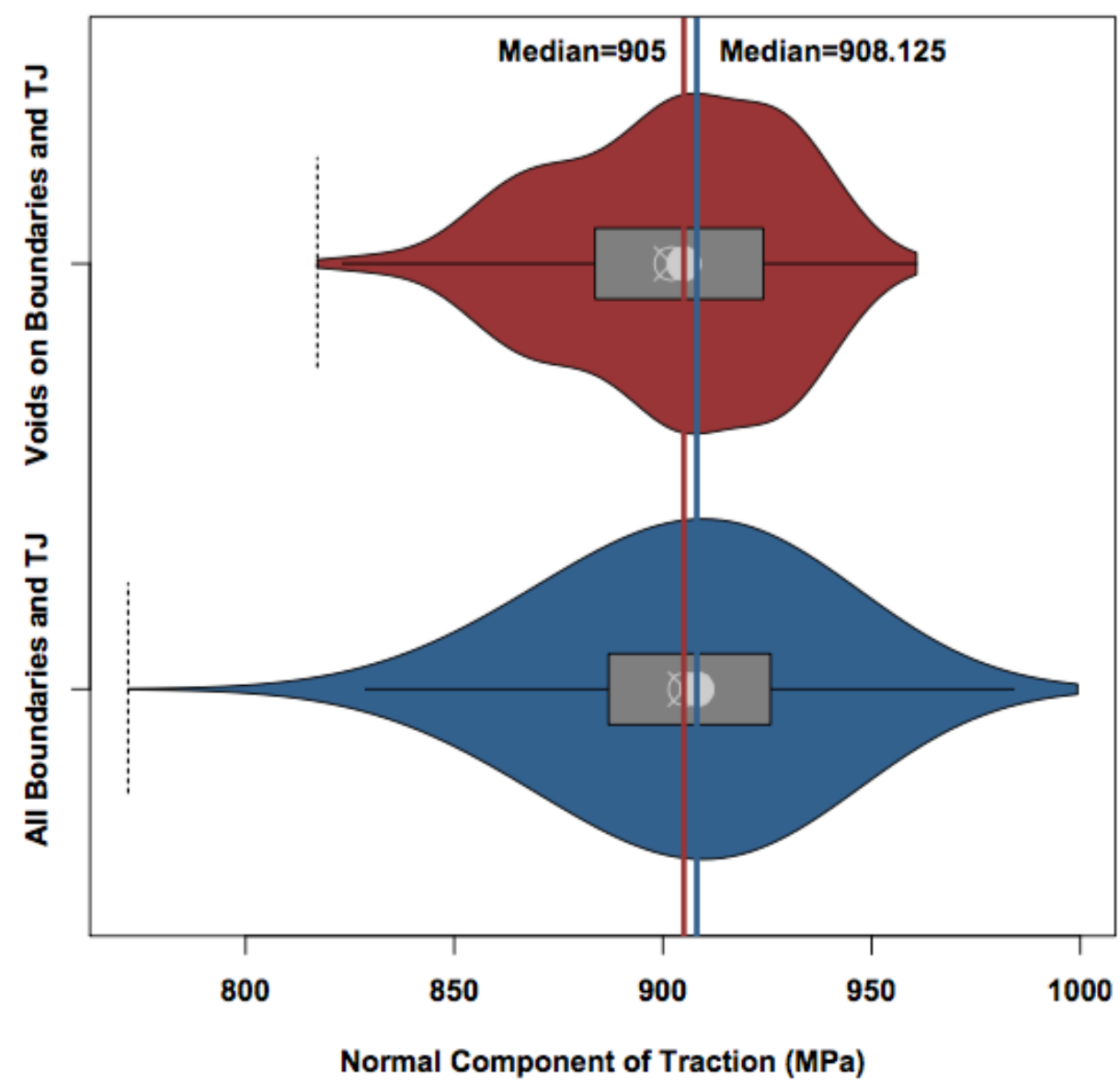

Figure 6: Violin plots comparing surface normal tractions for all grain boundaries and for the grain boundaries where voids are located. 


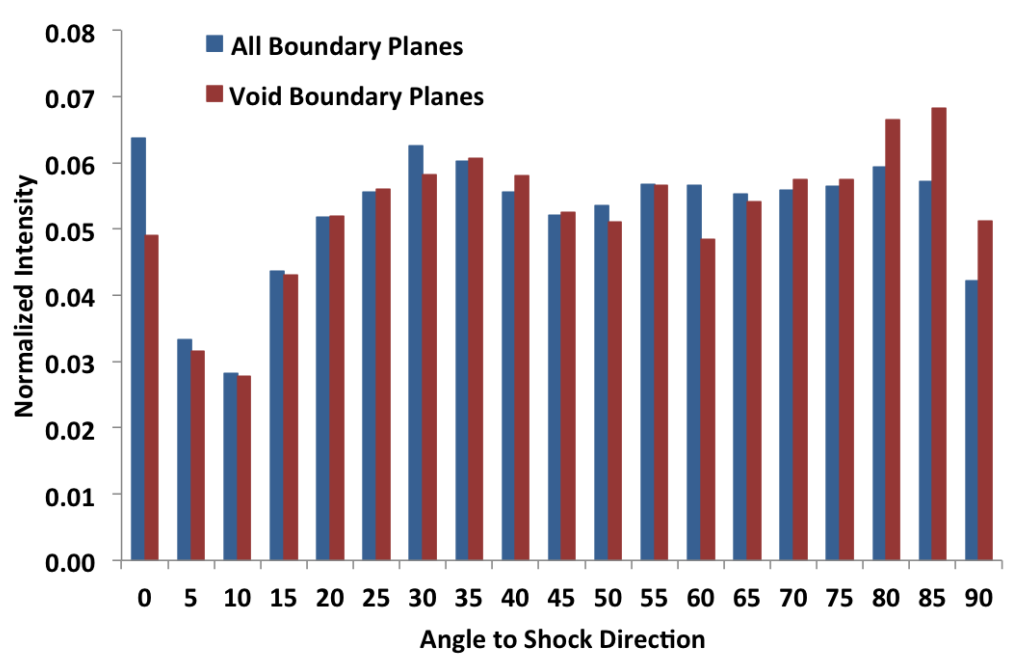

(a)

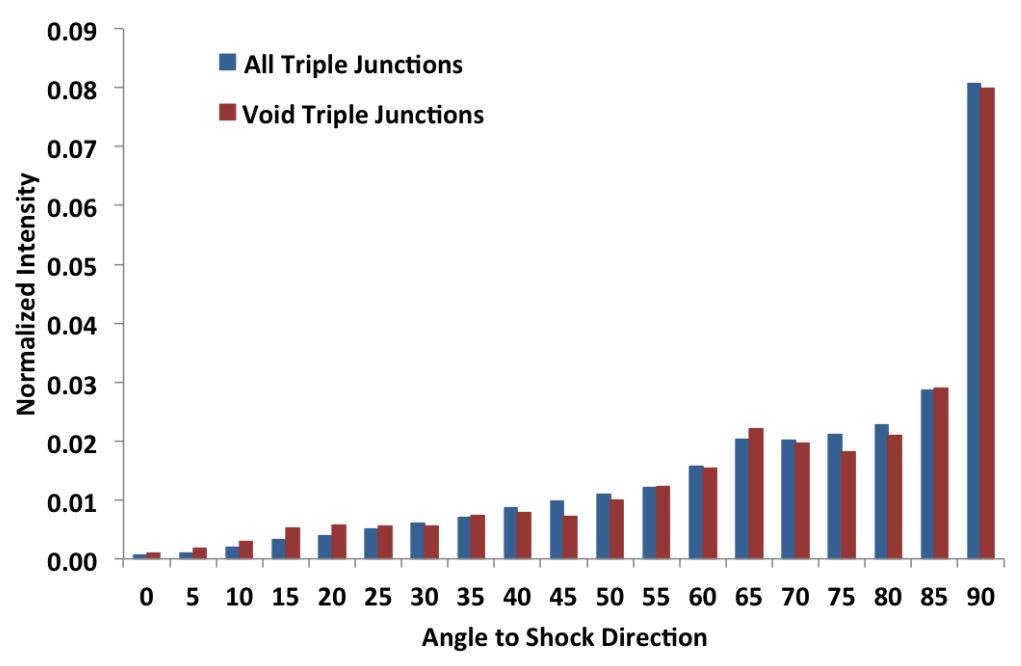

(b)

Figure 7: Comparison between all grain boundaries and grain boundaries developing porosity for: (a) the grain boundary plane normal inclination to shock direction and for (b) the triple junction tangent vector inclination to shock direction. 


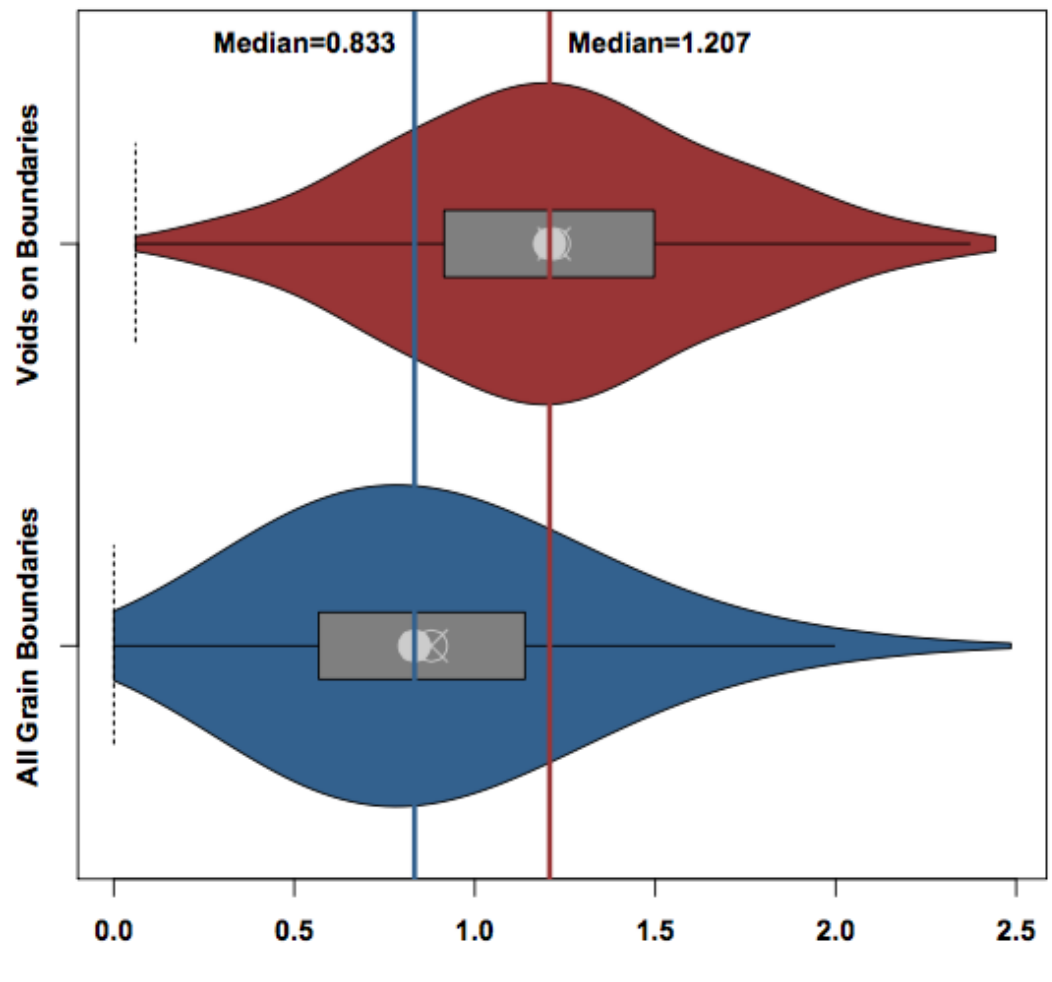

(a)

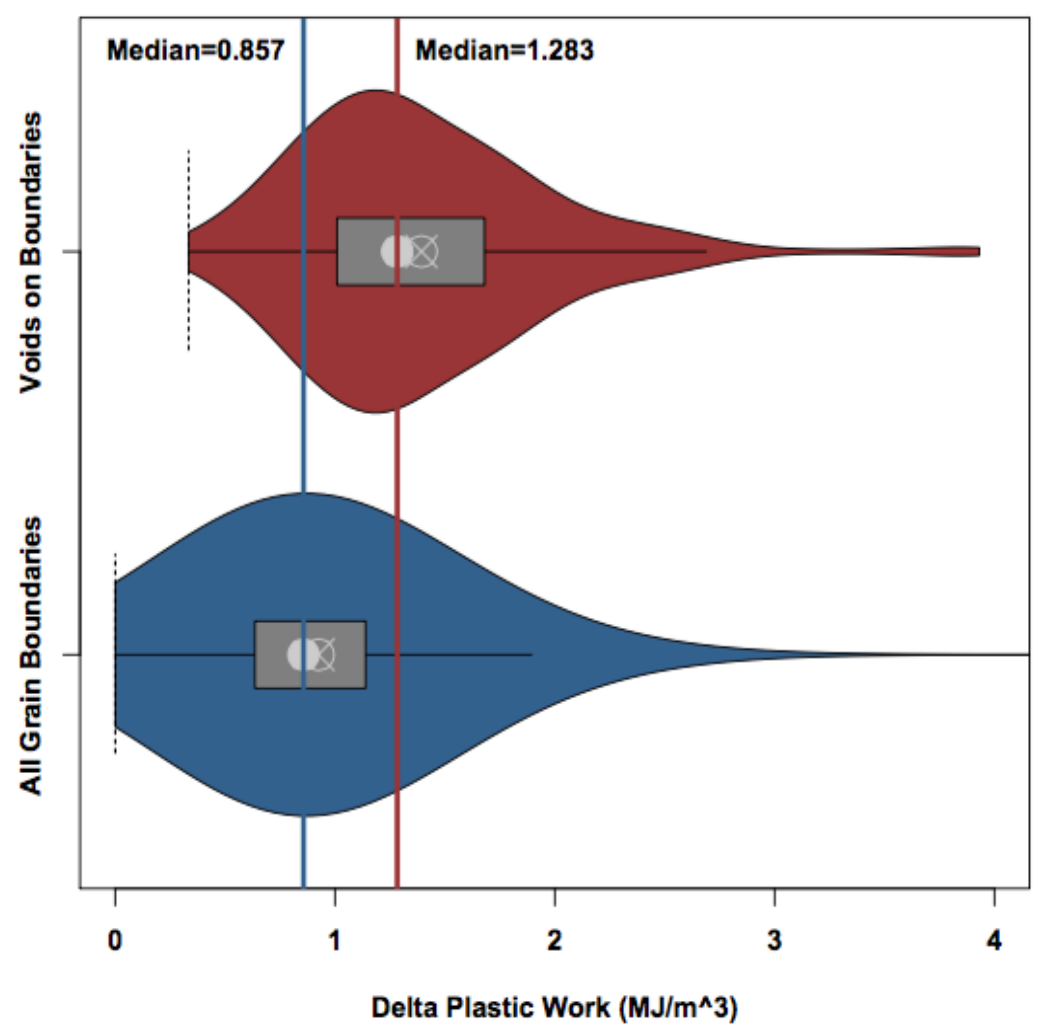

(b)

Figure 8: Violin plots of the distributions of (a) difference in Taylor factor and (b) difference in plastic work across a grain boundary for both the overall set of grain boundaries and for the grain boundaries at the void locations. 


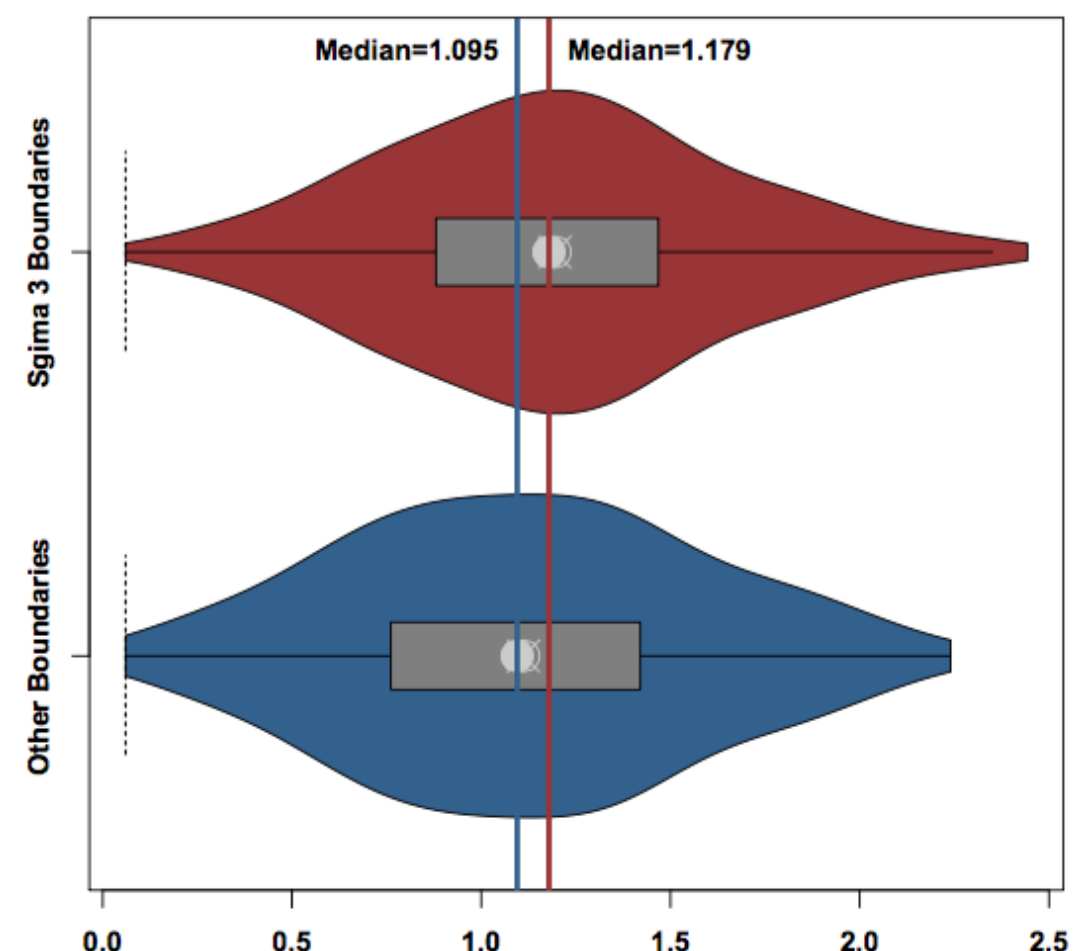

(a)

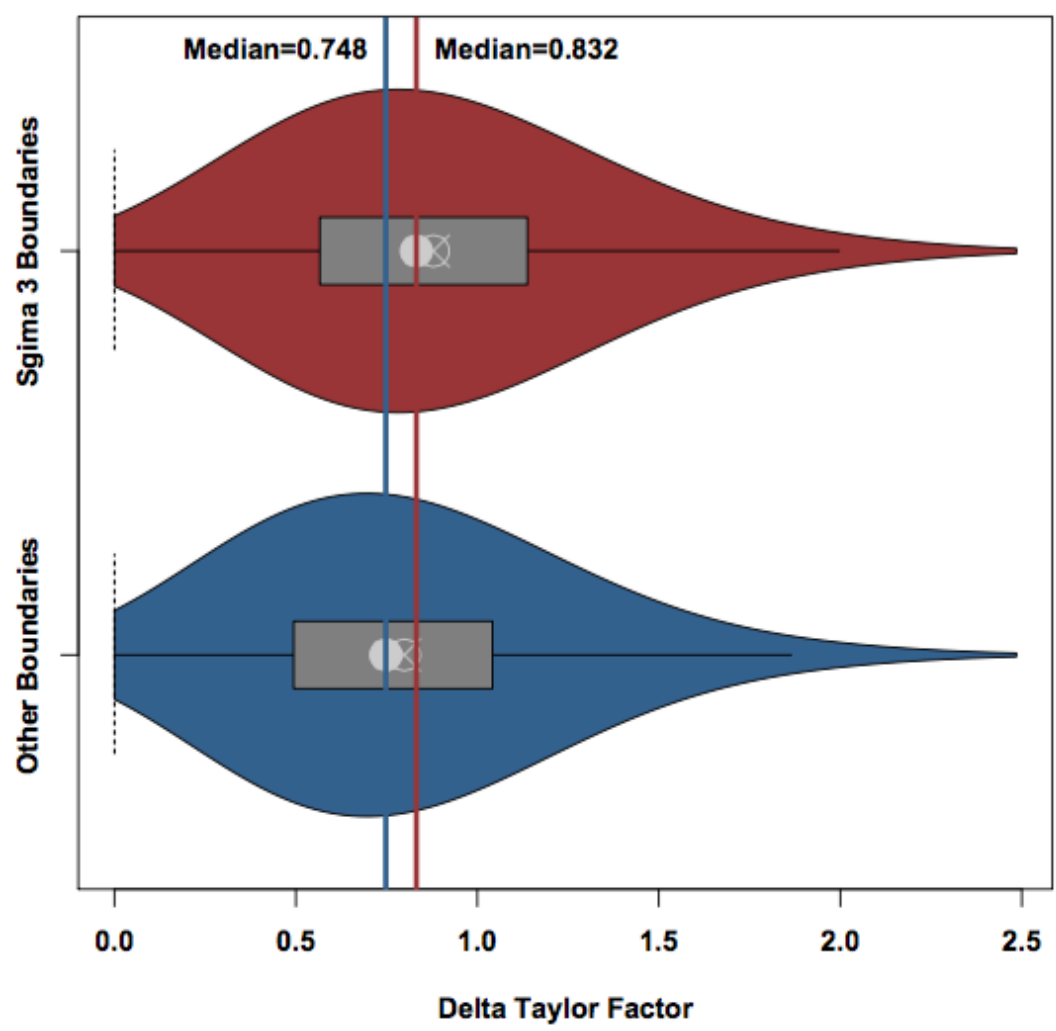

Figure 9: Violin plots for difference in Taylor factor partitioned by grain boundary type into sets for $\Sigma 3$ boundaries and all other boundaries and separately displayed for (a) the grain boundaries developing porosity and for (b) all grain boundaries. 

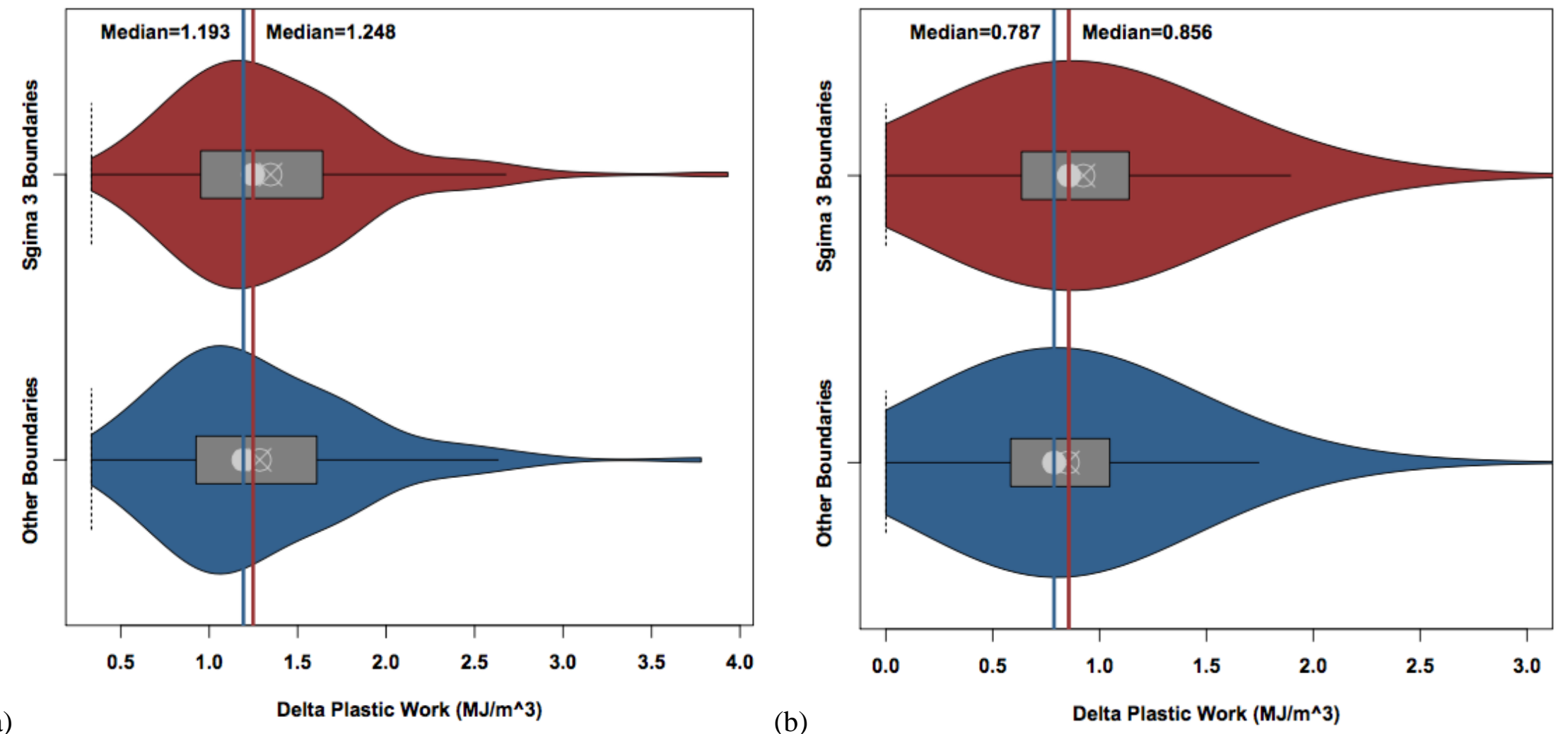

Figure 10: Violin plots for difference in plastic work partitioned by grain boundary type into sets for $\Sigma 3$ boundaries and all other boundaries and separately displayed for (a) the grain boundaries developing porosity and for (b) all grain boundaries. 


\begin{tabular}{|c|c|c|}
\hline Type of Interface & Menasche et al. & This work \\
\hline Total Voids & 485 & 447 \\
All Interface Voids & $332(68.5 \%)$ & $308(68.9 \%)$ \\
Grain Boundary Plane & $196(40.4 \%)$ & $156(34.9 \%)$ \\
Triple Junction & $126(26.0 \%)$ & $99(22.2 \%)$ \\
Higher-Order Interface & $10(2.1 \%)$ & $53(11.9 \%)$ \\
\hline
\end{tabular}

Table 1: Distribution of voids at grain boundary features from Menasche $e t$ al.$^{[32]}$ and from this work.

\begin{tabular}{|c|ccc|}
\hline Hellinger Distances & Combined & $\Sigma 3$ Boundaries & Other Boundaries \\
\hline Delta Taylor Factor & 0.276 & 0.287 & 0.262 \\
Delta Plastic Work & 0.323 & 0.334 & 0.313 \\
\hline
\end{tabular}

Table 2: Hellinger distances between full and restricted distributions by grain boundary character. 


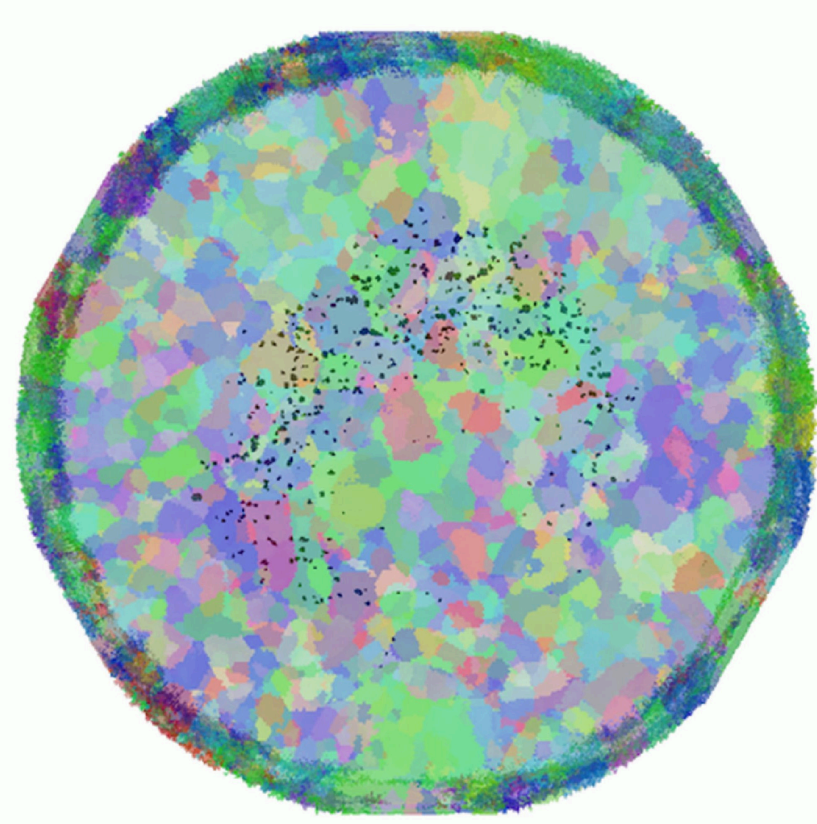

3-D orientation map of undeformed copper sample with void locations

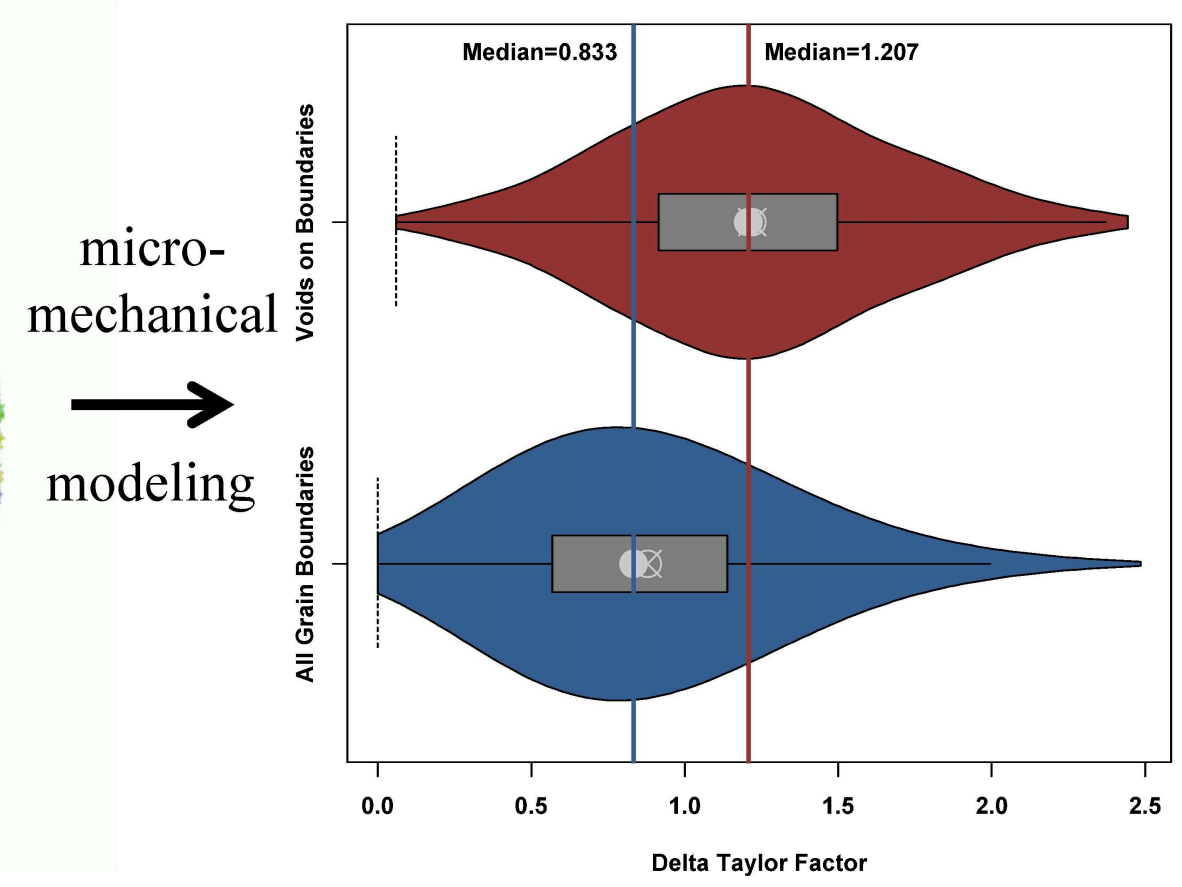

Statistical analysis of micromechanical fields to discover correlations with damage occurrence 